an unfavourable factor, being associated with a greater fall in ICAP during carotid clamping but, since most patients with this abnormality did accept carotid ligation without developing hemiplegia, the presence of this vascular anomaly was not a contraindication to carotid ligation but merely a warning that caution must be observed.

\section{DIFFERENTIAL DIAGNOSIS OF CEREBELLOPONTINE ANGLE LESIONS BY VERTEBRAL ANGIOGRAPHY}

LINDSAY SYMON and BRIAN KENDALL (London) had used vertebral angiography for the past five years as a definitive investigation for providing details of the sites and probable natures of cerebellopontine angle lesions. Advantages of angiography were its performance under basal sedation with consequent diminution of the disturbance of intracranial dynamics consequent on anaesthesia, and its potential use as an elective investigation not necessarily urgently followed by surgery. The angiograms of 141 cerebellopontine angle masses were reviewed and plain film changes were mentioned. Erosion of the internal auditory meatus was demonstrated in 63 of 78 patients with acoustic neuromas and in two of the 22 posterior fossa meningiomas. Sclerosis of the petrous bone was evident in five of these posterior fossa meningioma cases. The six trigeminal neuromas were associated with fairly typical apical petrous erosions with smooth edges. These findings were also present in one patient with a chordoma and in one with a meningioma. Plain radiographs of the skull were normal in 15 of the acoustic neuromas, 10 of the meningiomas, and in a variety of other less common conditions.

The importance of adequate study of the venous phases in angiography was emphasized. Small masses usually first displaced a complex of veins running towards the petrosal vein which passes from the lateral aspect of the pons to the superior petrosal sinus. The typical arterial displacements associated with acoustic neuroma and trigeminal neuroma were demonstrated. The occurrence of pathological circulation in two-thirds of the acoustic neuroma cases was emphasized, and the importance of adequate visualization of both external carotid and internal carotid circulations in the detection of pathological vessels in meningiomas and acoustic neuromas was emphasized. The common source for the pathological circulation in acoustic neuromas was found to be either the dural distribution of the vertebral supply or the meningeal or ascending pharyngeal branches from the external carotid circulation. The blood supply of the angle meningiomas usually emanated either from the hypophysial branch of the internal carotid artery or the external carotid circulation. The vertebral circulation sometimes, but not invariably, played a part.

INTERNAL CAROTID BIFURCATION ANEURYSMS

AND THEIR TREATMENT BY DIRECT SURGERY

R. P. SENGUPTA (Newcastle) described nine cases of aneurysm at the bifurcation of the internal carotid artery. All were submitted to direct intracranial surgery on the neck of the sac, and eight aneurysms were occluded by spring clips. No patient died and there was only one poor result. From an analysis of these cases and also from a review of the literature some of the characteristics of these aneurysms emerged. They accounted for about $7 \%$ of all intracranial aneurysms, appeared to be usually on the left side, and were equally distributed between the sexes. When associated with other aneurysms those at the carotid bifurcation were responsible for haemorrhage. Demonstrations of small aneurysms might be difficult, and accurate angiographic definition of the aneurysm neck might not be possible. The position of an internal carotid bifurcation aneurysm was either on the origin of the anterior cerebral artery or on the origin of the middle cerebral artery. With careful exposure and appropriate positioning of the head, it was possible to occlude these aneurysms? with clips. Carotid ligation might not prevent furthen haemorrhage in this group of aneurysms.

\section{RESULTS OF SURGICAL TREATMENT OF} PERICALLOSAL ANEURYSMS

D. G. T. THOMAS and A. PATERSON (Glasgow) reported a series of 36 cases of pericallosal aneurysms admitted for neurosurgical management between 1960 and 1974. Direct clipping of the aneurysm had been performed in 30 patients with an operative mortality of $23 \%$ (seven deaths). A further seven $(23 \%$ ) had considerable disability, but $54 \%$ (16) did well. Two patients were excluded from surgery and four died before operation as a result of recurrent haemorrhage at two, six, eight, and 16 days after the initial bleed. Preoperative clinical grading, age, and sex did not correlate with the outcome. Fifteen patients $(50 \%)$ were found at operation to have significant haematomas, but the results of treatment were no worse in these cases, and five of them before operation were alert without focal signs. Surgery within the first week of haemorrhage was not associated with an increased mortality and was considered to have forestalled death from recurrent haemorrhage. The results of previous series were reviewed and the evidence was considered to indicate that pericallosal aneurysms had a rather poor prognosis, although operative treatment was superior to conservative methods. 